\title{
Design on the System of Equipment Material Support Internet of Things
}

\author{
Zhang Donghu ${ }^{1, a}$ Wang Tiening ${ }^{1, b}$ Niu Xingxing ${ }^{1, c}$ \\ Yu Shuangshuang ${ }^{1, d}$ \\ ${ }^{1}$ Academy of Armored Forces Engineering, Beijing, China \\ alannimi@sina.com, byuamanda@126.com, c490794736@qq.com, ${ }^{d} 574137379 @ q q . c o m$
}

Key words: Internet of Things (IOT); equipment material support; support capability

\begin{abstract}
The Internet of Things (IOT) plays an important role in improving the equipment support capability. Using the Internet of Things, cloud computing and other new advanced information technologies, the equipment material support system is constructed. In this paper, the architecture, the network structure and building steps of the equipment material support internet of things system are designed to promote the development of equipment material support.
\end{abstract}

\section{Introduction}

The Internet of Things (IOT) has been widespread concerned for that it is the key scientific research project to tackle key problems and it is the information technology tide following the Internet and new economic engine of the world in next period. As an important part of our military equipment support, the equipment material support can improve its support capability with the organic integration of the IOT.

\section{The Architecture of the Equipment Material Support Internet of Things System}

The Internet of Things means that things and things or people and things or people and people are connected with each other by the technology. It is the network connecting any article with the Internet through RFID, 2D bar code, electronic tag, infrared sensor, global positioning system, laser scanner and some other information technologies in order to realize intelligent identification, positioning, tracking, monitoring, precise management and real-time control.

The equipment material support internet of things system brings the conception of the internet of things into the equipment material support. With the platform of IOT, it achieves high efficient operation in the information system. As shown in Fig. 1in next page, we can see that the system consists of three layers, which are the perception layer, the network layer and the application layer.

(1) Perception layer

The function of the perception layer is to realize the intelligent perception and identification, the information acquisition and processing, the automatic control on the equipment materials. Through the communication terminal modules it connects the material entities to the network, mainly including various sensors such as infrared sensors, photoelectric sensors, distance sensors, RFID tags, bar codes, smart devices, electronic tags, GPS modules and other relating devices and technologies. It adopts different modes of perception for the business demands and characteristics in different periods of the equipment support. For example, using RFID or 2D bar codes we can get the variety, quantity and cargo space information of the material in the storage process. Using GPS, quality testing devices and wireless network communication we can obtain the location and quality information of the materials in the supply process. 


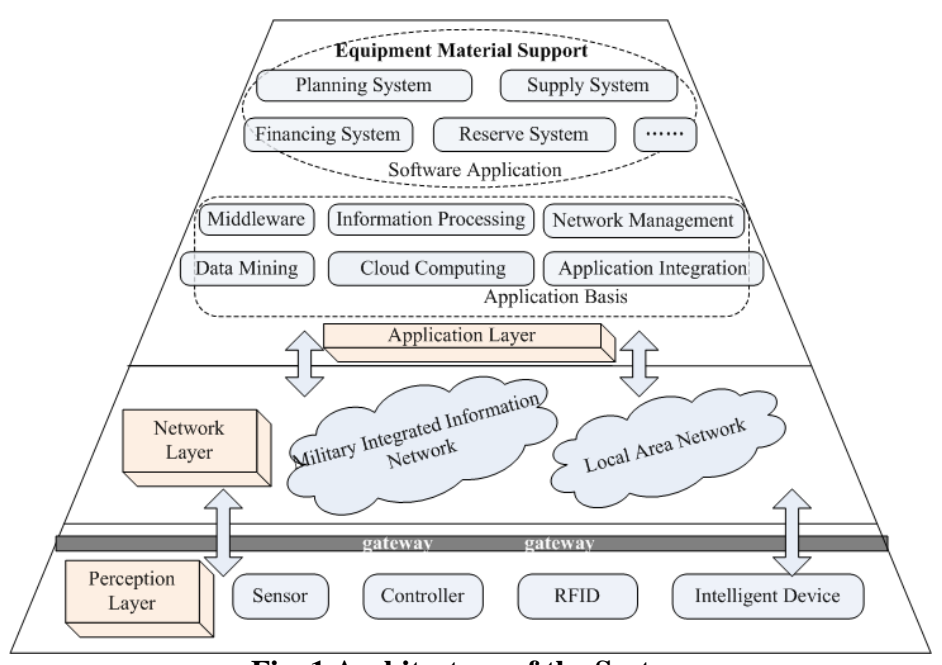

Fig. 1 Architecture of the System

(2) Network layer

The network layer mainly consists of the Access Network and the Core Network, the wired and wireless network, the WAN and LAN to realize the transmission, sharing and control of the information. The equipment material support internet of things can transfer information via the military integrated information network or the close-range wireless network technology such as Bluetooth, Wifi, and ZigBee. The network layer is required to have safe and reliable gateway technology as well as communication nodes and networking technologies with high speed, low power and short distance in the perception layer.

(3) Application layer

The application layer is made up of two layers: the application basis layer and the software application layer. The application basis layer is the foundation of the software application layer. Using the basic data processing, application integration, cloud computing, data mining, middleware and network management it classifies the information into three kinds--basic data, business data and decision-making data. It sets the access rights and determines the information sharing degree. With the cloud computing platform it achieves high efficient use of resources and high-performance computing capability. Through data mining technology it achieves real analysis on the information to ensure that the decisions are right. The software application layer is an information system terminal oriented to users to establish modular integrated information systems, and fully integrate the business functions of equipment material support. Then establish the equipment material planning subsystem, the financing subsystem, the reserve subsystem, the supply subsystem respectively, and achieve the human-computer interaction in $\mathrm{B} / \mathrm{S}$ or $\mathrm{C} / \mathrm{S}$ mode.

\section{Network Structure of the Equipment Material Support Internet of Things System}

The equipment material support internet of things system is built on the basis of the internet of things and cloud computing platform. The network structure schematic of the system is shown in Fig. 2 in next page.

During the material order period, obtain real-time information such as the production progress information, the quality testing information, the dynamic information of the suppliers via the Internet, summarize the production information to the headquarter web server, and establish the e-commerce system for the material order tasks via the headquarter web server. Transfer the data in the server to the material order center through the network separator, and realize safe interoperability of the information between the Internet and the military inner network. 


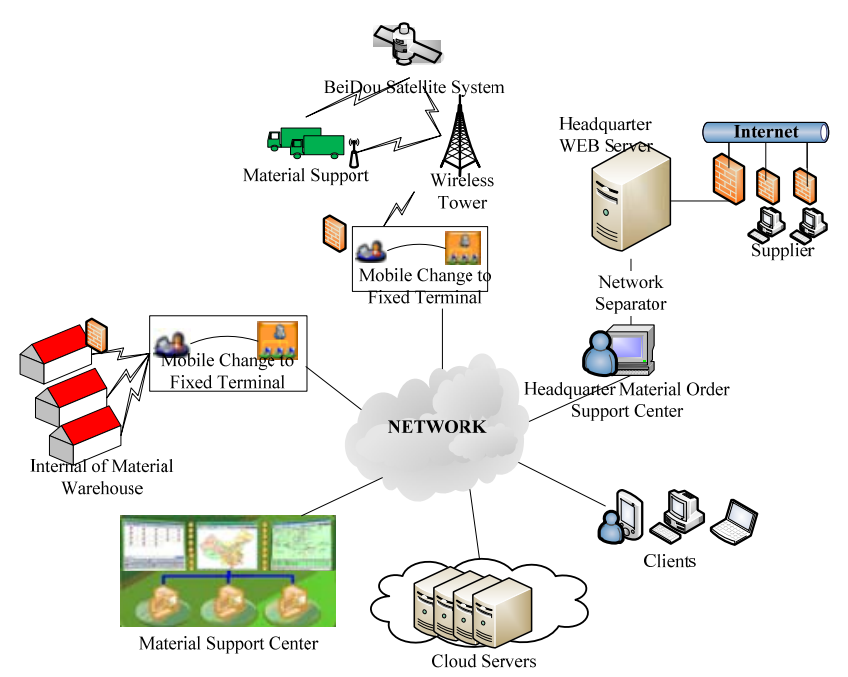

Fig. 2 Network Structure Schematic of the System

During the material storage period, due to the small storage space, the frequent activities and the diverse data exchange in the warehouse, establish the wireless local area network to make quick perception and control of the information, so that the warehouse business can be easily put into effect. Due to the long transmission distance of the information externally, the material warehouse change the wireless network information into the military integrated network information via TCP/IP network protocol. On the one hand, the wired transmission has better anti-stolen and anti-disruption capacity than the wireless transmission. On the other hand, the wired transmission can increase the speed of information transmission.

In the material supply process, as the range of material transporting is large and the location of the material is not fixed, we have to adopt the wireless communication transmission mode. The materials in transporting are equipped with GPS positioning system and wireless transmission devices, and with the help of the BeiDou satellite system and the wireless receiver tower we can monitor the whole transporting process in real time. In addition, after receiving the data of each period, only the receiving data, distribution data and the system in the warehouse are stored and operated in the inner server of the warehouse while the other data and information systems are all saved in the cloud server, so that the integrated management, resources sharing and high efficient processing can be carried out. The equipment material support information center can implement effective management and security protection on the cloud information. The users having permissions apply the cloud information system via the terminals such as PCs, desktops, laptops, etc. They get the information from the cloud and control it by the equipment material support internet of things.

\section{Building Steps of the Equipment Material Support Internet of Things System}

The building of the system is a huge project. In order to complete the project perfectly, it should be initiated mainly from the following aspects.

The first step is completing the top design. In this stage, the needs of the system and the main process information are determined in view of the system integration, and modules classification to indicate the direction.

The second step is establishing the system platform and the network structures.

The third step is building the hardware and software infrastructures, such as standard programming standards, set interface standard, construct basic database, connect various needed sensors.

The fourth step is selecting a breakthrough, classifying modules and developing parallel. The system involves many modules, and at the same time, the construction is not in conformity with the support ability construction principles and strategies. The material storage capacity is concentrated, and easy to make effect. As a result, the storage system should be constructed at first in different modules. 
The fifth step is module integration and test. After the completion of each module, through the interface and middleware technologies the modules are merged together. Then the initial material storage system is tested in various aspects to find the insufficiency.

The sixth step is trying to run the entire system, firstly in some regions gradually, such as single supply chain. The pilot but must have all the modules of the equipment support. In personnel training, through the commission, check and improve the function of the system.

The last step is the comprehensive promotion, management and maintenance. After a certain period commission, it's time to start to promote the use of the system and do some management and maintenance, also solve the issues from each department.

\section{Conclusions}

The equipment material support internet of things system is a complex system. There are still some problems need to be solved, of which the most important one is the network safety. We should pay more attention to the safe and secret problems, and take effective actions to establish more perfect system based on IOT to improve the automatic and intelligent level of our military equipment support capability.

\section{References}

[1] Jingxi Tian. The Outline of Internet of Things [M]. Nanjing: Southeast University Press, 2010: $1-8$.

[2] Tiening Wang. Strategic armored equipment material support engineering [M]. Beijing: Weapon Industry Press, 2006: 7-10.

[3] Zongxi Wang. Military logistics [M]. Beijing: Tsinghua University Press, 2007: 10-12.

[4] Chuan Song, Consideration on the construction of internet of things in communication commands equipments [J]. Military communication academy, 2010: 15-17.

[5] Gustavo Ramirez Gonzalez, Early Infrastructure of an Internet of Things in Spaces for Learning[C]. 2008 IEEE International Conference on Advance Learning Technologies. IEEE Press, 2008:300-303 